\title{
Development of a 3D-printed external ventricular drain placement simulator: technical note
}

\author{
Bruce L. Tai, PhD, ${ }^{1,2}$ Deborah Rooney, PhD, ${ }^{3}$ Francesca Stephenson, BS, ${ }^{1}$ Peng-Siang Liao, BS, ${ }^{4}$ \\ Oren Sagher, MD, ${ }^{2}$ Albert J. Shih, PhD, ${ }^{1}$ and Luis E. Savastano, MD²
} Departments of ${ }^{1}$ Mechanical Engineering, ${ }^{2}$ Neurosurgery, and ${ }^{3}$ Learning Health Sciences, University of Michigan, Ann Arbor,
Michigan; and ${ }^{4}$ Advanced Institute of Manufacturing with High-Tech Innovations, National Chung Cheng University, Taiwan

\begin{abstract}
In this paper, the authors present a physical model developed to simulate accurate external ventricular drain (EVD) placement with realistic haptic and visual feedbacks to serve as a platform for complete procedural training. Insertion of an EVD via ventriculostomy is a common neurosurgical procedure used to monitor intracranial pressures and/or drain CSF. Currently, realistic training tools are scarce and mainly limited to virtual reality simulation systems. The use of $3 \mathrm{D}$ printing technology enables the development of realistic anatomical structures and customized design for physical simulators. In this study, the authors used the advantages of 3D printing to directly build the model geometry from stealth head CT scans and build a phantom brain mold based on 3D scans of a plastinated human brain. The resultant simulator provides realistic haptic feedback during a procedure, with visualization of catheter trajectory and fluid drainage. A multiinstitutional survey was also used to prove content validity of the simulator. With minor refinement, this simulator is expected to be a cost-effective tool for training neurosurgical residents in EVD placement.
\end{abstract}

http://thejns.org/doi/abs/10.3171/2014.12.JNS141867

KEY WORDS external ventricular drain model; simulator; ventriculostomy; surgical technique; intracranial pressure; virtual reality

$\mathrm{I}$ NSERTION of an external ventricular drain (EVD), or ventriculostomy, is one of the most common neurosurgical procedures, often performed under emergency conditions in intensive care units or trauma centers. Insertion of an EVD is indicated for intracranial pressure (ICP) monitoring or CSF drainage in patients with traumatic brain injury, hemorrhagic stroke, or hydrocephalus. It is often the first neurosurgical procedure that trainees are required to learn and master during residency. The learning curve to perform this surgical procedure is relatively steep. Novice residents may have difficulty appropriately performing the sequence of steps skin-to-skin and may require several catheter passages though the brain before puncturing the ventricle, often leading to higher complications rates. ${ }^{11}$ According to recent publications, hemorrhagic complication rates of EVD placement can be as high as $30 \%-40 \%,{ }^{15}$ and documented EVD-related infections have also been reported in as many as $20 \%$ of cases..$^{7,10}$ The high volume of procedures performed every year increases the risk profile of EVD placement, increasing the need for an effective surgical training tool and the need to standardize the evaluation of neurosurgeons who perform this procedure. ${ }^{1}$ EVD placement is generally performed in emergency situations at unpredictable times, and residents must become proficient early in their training. ${ }^{3}$ A study by Huyette et al. ${ }^{11}$ measured the accuracy of freehand catheter placement of EVDs and found that $22 \%$ of placement attempts landed the catheter in nonventricular spaces, even after repeated insertions. Repeated attempts can traumatize brain tissue and lead to complications such as infection and hemorrhage. ${ }^{9,20}$ A study of patient complications associated with EVD placement found that the most serious complications occurred when the surgeon was inexperienced. ${ }^{22}$

\footnotetext{
ABBREVIATIONS EVD = external ventricular drain; ICP = intracranial pressure; $V R$ = virtual reality.

SUBMITTED August 12, 2014. ACCEPTED December 11, 2014.

INCLUDE WHEN CITING Published online June 26, 2015; DOI: 10.3171/2014.12.JNS141867.

DISCLOSURE Funding for this project was received from the Blue Cross Blue Shield Michigan Foundation (A.J.S., B.L.T., D.R., and O.S.), and the Clinical Simulation

Center at the University of Michigan (B.L.T.).
} 
Neurosurgical residents have few options for practicing EVD placement. Historically, surgical residents have been trained by participating in as many surgeries as possible on live patients while under close supervision. However, although not exclusively for EVD, studies have shown that intraoperative training could often result in longer operation times, higher financial costs, and greater risk of complications. ${ }^{5,17,19,26}$ Cadavers are not a viable option for EVD placement, because preservation techniques change the tactile properties of brain tissue and the ventricular system tends to be distorted or collapsed, making cadavers an inadequate substitute for a live patient. Using simulators for surgical training allows residents to develop the necessary motor skills safely and realistically, without impacting patient care. ${ }^{6}$ Most EVD simulators currently on the market are virtual reality (VR)-based. One of the widely used commercial products was developed by Luciano et al. ${ }^{14}$ That system includes a high-resolution stereoscopic display and a haptic device with head- and hand-tracking capabilities. The user wears stereoscopic goggles that track head movement and adjusts the virtual display accordingly. The user operates on the virtual patient with a haptic stylus in one hand and defines the cutting plane with a tool held in the other hand. The haptic scalpel offers tactile feedback according to the physical characteristics of the tissue being cut; this feature allows the user to get a feel for the different textures of the skin, brain, and bone. Although the VR systems have been validated by neurosurgical residents,, 313 they are still often limited in their ability to mimic the physical properties or phenomena of EVD placement, such as sidewall collisions and limited range of motion..$^{12}$ In addition, these systems were developed to train the operator on catheter trajectory, leaving aside the other key steps of the procedure. Widespread availability is another major concern for VR simulators, because these devices often cost tens to hundreds of thousands of US dollars. ${ }^{21}$

For low-cost, high-fidelity simulation to practice EVD placement skin-to-skin, we designed a physical modelbased simulator using 3D printing from a stealth head CT scan of a de-identified patient. Three-dimensional printing is a rapidly emerging prototyping technology that enables 3D fabrication with high resolution and customized design. Engineers and surgeons can therefore collaborate to develop the simulator based on clinical needs. The physical simulator provides the same anatomical accuracy as a VR simulator but overcomes the mechanical and procedural limitations. A computer-based simulator adds a level of difficulty for students, as the computer forces the student to translate 3D operating space into an inauthentic 2D space. A physical simulator exists in the "real world" and thus can be used with real surgical tools to create a realistic, immersive simulation environment. In this environment, participants can more easily suspend their disbelief, creating a highly effective learning environment. ${ }^{6}$ The lessons learned can be perceived as more realistic in comparison with simpler screen-based simulators. ${ }^{18}$ There exists a 3Dprinted simulator for neurosurgery using multimaterial printers, and it has demonstrated the value and feasibility of using a physical model in training., ${ }^{4,25}$ However, despite the accurate anatomy produced by this method, material selection is limited by the machine and current printing technologies and thus could be inadequate to make realis- tic phantom tissues. In this study, we used both 3D printing and traditional molding technologies, along with phantom materials, to emphasize the model's anatomy, tactile feedback, functionalities, and overall durability, aiming for a high-fidelity simulator. We highlight the design of important features needed in EVD training and the process used to evaluate preliminary validity evidence for the simulator.

\section{Methods \\ Simulator Design}

Figure 1 depicts the design of the simulator, which consists of a skull frame, skull cap, replaceable insert, and brain phantom. The skull cap and frame are secured via 3 tabs. Within the skull cavity, the brain phantom is held against a transparent plastic plate in the midline of the skull, allowing for observation and evaluation of catheter placement. The skull cap contains a disposable insert, 40 $\mathrm{mm}$ wide and $80 \mathrm{~mm}$ long, covering the potential operative region on the skull. This insert is for 1-time use and has an artificial scalp on top of it. The insert size is designed for anatomical accuracy and structural stiffness against the drilling load, while allowing the user some degree of freedom in site selection. The cavity containing the brain is filled with water to mimic pressure conditions inside the human skull. Pressure is controlled by the water reservoir suspended $20 \mathrm{~cm}$ above the ventricle, and the water fills the ventricle directly.

The skull frame and cap are manufactured with acrylonitrile butadiene styrene (ABS) plastic using 3D printing. The geometry of the model originates from CT scans of a de-identified patient and is modified to include fastening features using computer-aided design software (Mimics and Magics by Materialise). The skull cavity is made to accommodate a phantom brain. The brain is fabricated using a 3D-printed mold based on 3D scans of a plastinated human brain. To create a realistic texture, the phantom brain is molded with high-acyl gellan gum, a gelatin-like material chosen because of its high gelling efficiency and its ability to mimic the biomechanical properties of human brain tissue. ${ }^{8}$ The manufactured brain phantom and mold are shown in Fig. 2.

The skull cap insert is disposable and intended for 1-time use, and it can simulate a bur scalp incision, holedrilling, dura mater opening, skin closure, and catheter tunneling and anchoring. The insert geometry is extracted from the designated region in the skull cap (Fig. 1) and is $3 \mathrm{D}$ printed with plaster composite using a powder-bed machine. The insert is then treated with high-strength epoxy resin on the top and bottom to create a bicortical characteristic, followed by a thin layer of artificial galea and dura, and finally a 4-mm-thick layer of silicone scalp on the exterior surface (Fig. 3).

Figure 4 shows a photograph of the complete simulator setup. The skull is placed on a $30^{\circ}$ inclined ramp to mimic the patient's position during an actual EVD placement. The skull is connected to a water reservoir through a fluid input connector at its base. The simulator's water reservoir is affixed to a column adjustable to variable heights $(0-30$ $\mathrm{cm}$ above the ventricle) depending on the ICP needed in the different clinical scenarios. Inside the skull cavity, a fluid input tube directs the water into the lateral ventricle 


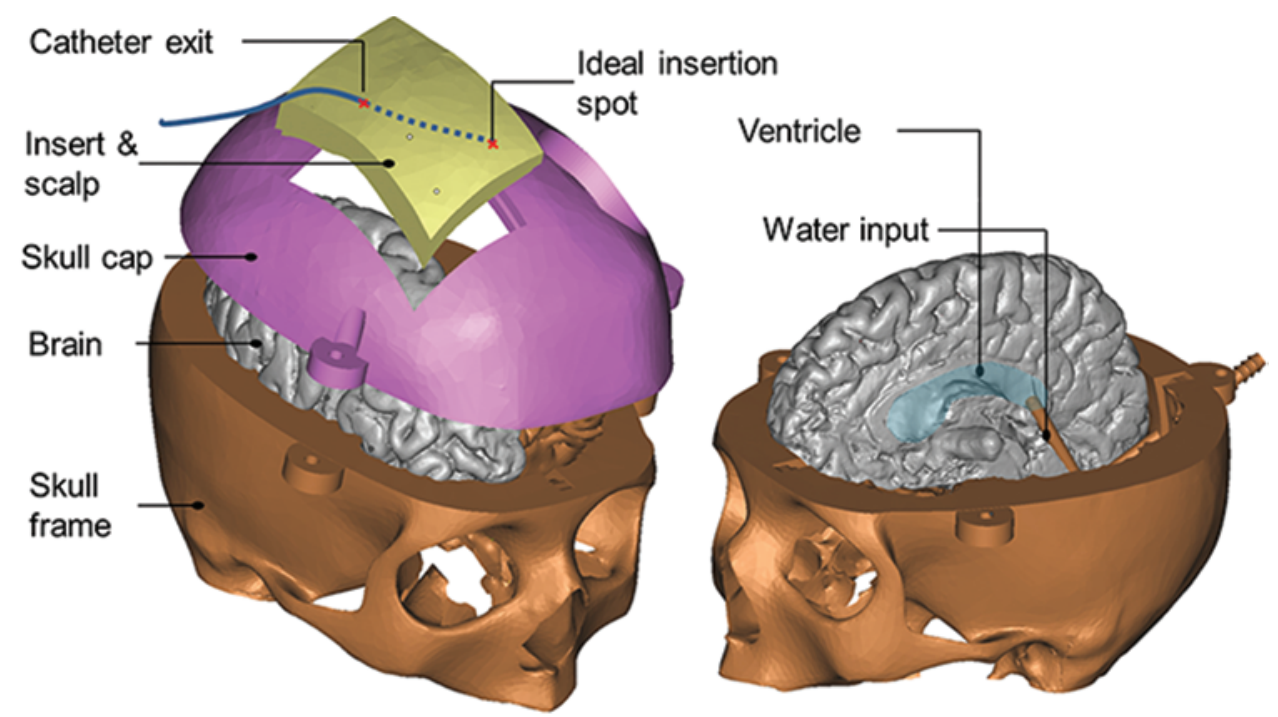

FIG. 1. Design of the EVD simulator based on real anatomy. Figure is available in color online only.

of the phantom brain (Fig. 1). Water fills the ventricle, creating pressure inside, allowing for a subtle "pop" sensation when the catheter penetrates the ventricle. On the other side of the skull cap are 2 open windows to observe the ventricle and catheter trajectory (Fig. 4). This feature allows users to correct improper insertion, particularly after multiple attempts.

\section{Preliminary Validation}

The preliminary validation process consisted of evaluation of specific evidence (content validity) and was aligned with best practices defined by the current "Standards" framework offered by the American Educational Research Association, American Psychological Association, and National Council on Measurement in Education. ${ }^{2}$

Following finalization of the simulator prototype, a sample of neurosurgeons from 3 Michigan-based neurosurgical training programs (University of Michigan, Wayne State University, and Henry Ford Hospital) evaluated the quality of the simulator at their own institutions.
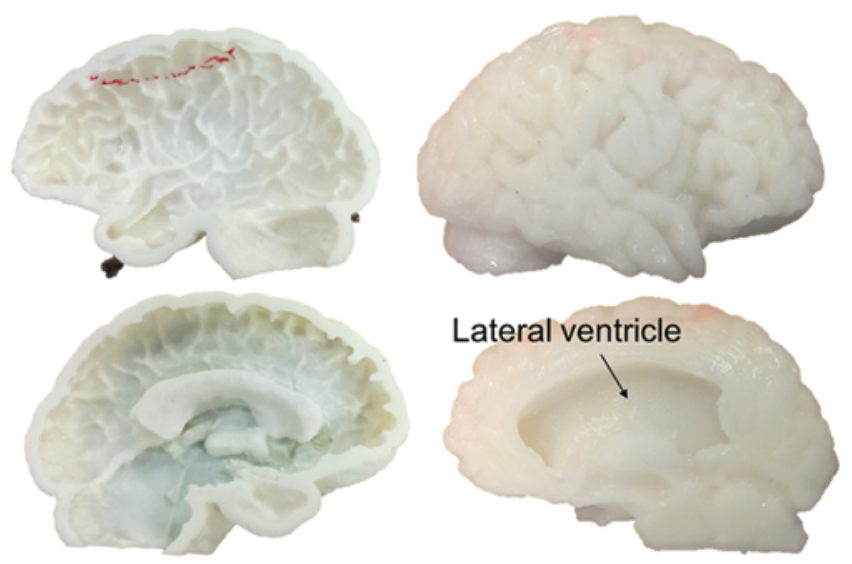

FIG. 2. The phantom brain used in the simulator is manufactured using a 3D-printed mold (left) filled with gellan gum (right). Figure is available in color online only.
Following their placement of an EVD on the simulator, each participant completed a 37-item survey rated on a 4-point rating scale, with 4 being the highest score. A sample of the targeted items from the survey is included in Table 1, in which each point refers to a certain subjective judgment on a specific aspect of the simulator. Based on best practices defined by Seagull and Rooney, ${ }^{23} 19$ items of the survey were targeted to measure experienced surgeons' opinions regarding the simulator characteristics and features and were organized across 4 domains: physical attributes, realism of experience, value of simulator, and a global (overall) rating. Participant responses were reported as frequencies, with group rating differences analyzed using a many-facet Rasch model. Inter-item reliability of the 19 targeted items was estimated using the Cronbach $\alpha$ test.

\section{Results}

Based on the design described above, a simulator prototype along with many inserts and phantom brains was built for evaluation. The simulator is designed to perform skin-to-skin EVD placement, by which participants would have a chance to practice the entire procedure, including tunneling and suturing (Fig. 5). A total of 17 surgeons (12 residents, 1 fellow, and 4 faculty neurosurgeons) from the 3 Michigan-based neurosurgical training programs evaluated preliminary validity evidence of the simulator. Overall, surgeon evaluator responses showed average ratings

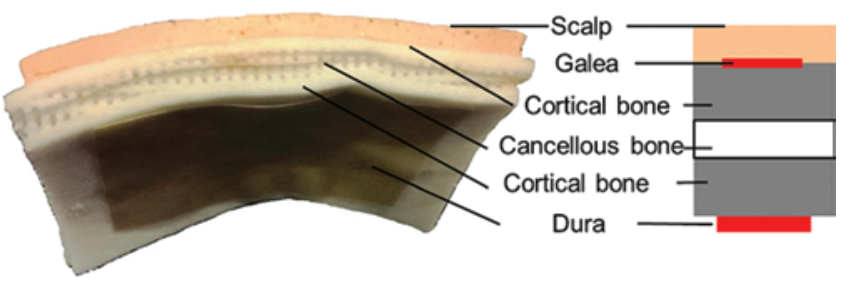

FIG. 3. The replaceable skull cap insert includes multiple layers to mimic the human skull. Figure is available in color online only. 

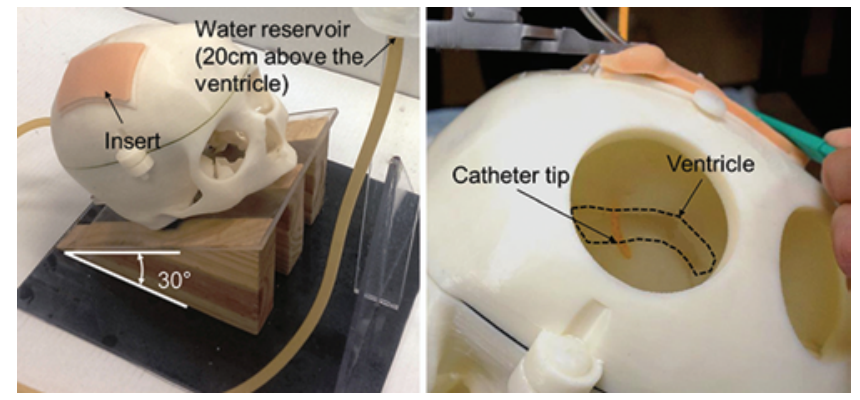

FIG. 4. Overall EVD model setup, including the inclined ramp, skull insert, and water reservoir (left), and windows to observe catheter trajectory (right). Figure is available in color online only.

of 3.4, 3.3, 3.9, and 2.5 (on a 4-point scale) for physical attributes, realism of experience, value of the simulator as a training tool, and global (overall) domains, respectively. The highest score of 3.9 was obtained for "value of simulator as a training tool for novice neurosurgeons," indicating this model had high potential for serving as a training tool. The survey details, corresponding scores, and comments are listed in Table 2. Faculty evaluators had higher overall averaged scores $($ mean $=3.4)$ than the fellow (mean $=3.3$ ) or residents (mean $=3.0 ; \mathrm{p}<0.05)$. A deeper analysis indicated no rating differences at the item level $(p>0.05)$. Six $(35.3 \%)$ of 17 evaluators indicated the simulator was useful in its current form and indicated, "This simulator can be used in training ventriculostomy placement as is, but could be improved slightly." Ten (58.8\%) of 17 evaluators indicated, "This simulator requires minor adjustments before it can be considered for use in ventriculostomy place- ment training." The Cronbach $\alpha$ for the targeted items was estimated to be high (19 items, $\alpha=0.90$ ).

\section{Discussion}

The use of 3D printing technology in the field of surgical simulation has led to many innovations, particularly for customized design and patient-specific simulation. Along with molding techniques to shape tissue-mimicking phantoms, physical models are expected to be more practical than VR simulators. In the particular case of EVD placement simulators, VR models focus on trajectory training, while our physical model was designed and developed as a comprehensive training tool for all the steps involved in EVD placement. Specifically, our model allows identification of surface anatomy and skin incision, skin retraction, bur-hole drilling, sharp opening of dura, catheter passage into the brain, skin closure, and subcutaneous catheter tunneling and catheter anchoring. One major advantage of our model over the existing products is that it provides immediate realistic visual feedback of EVD placement by observing CSF flow in the proximal end of the catheter and allows troubleshooting during various steps of the procedure, imitating (with high fidelity) problems found in "real-world" scenarios, such as: lack of CSF flow caused by malposition of the catheter, air locks, and a low-pressure system; occlusion of the catheter by debris; excessively tight anchoring stitches; and damage to the catheter while suturing skin. In addition, skull windows in the left hemicranium offer real-time visualization of the position and trajectory of the catheter within the intracranial compartment, with realistic depth and trajectory range through the bur hole. The simulator also allows

TABLE 1. Example of survey form for evaluating ventriculostomy simulator validity

\section{VENTRICULOSTOMY MODEL EVALUATION}

\section{Evaluator Name:}

Approximately how many ventriculostomy procedures have you performed?

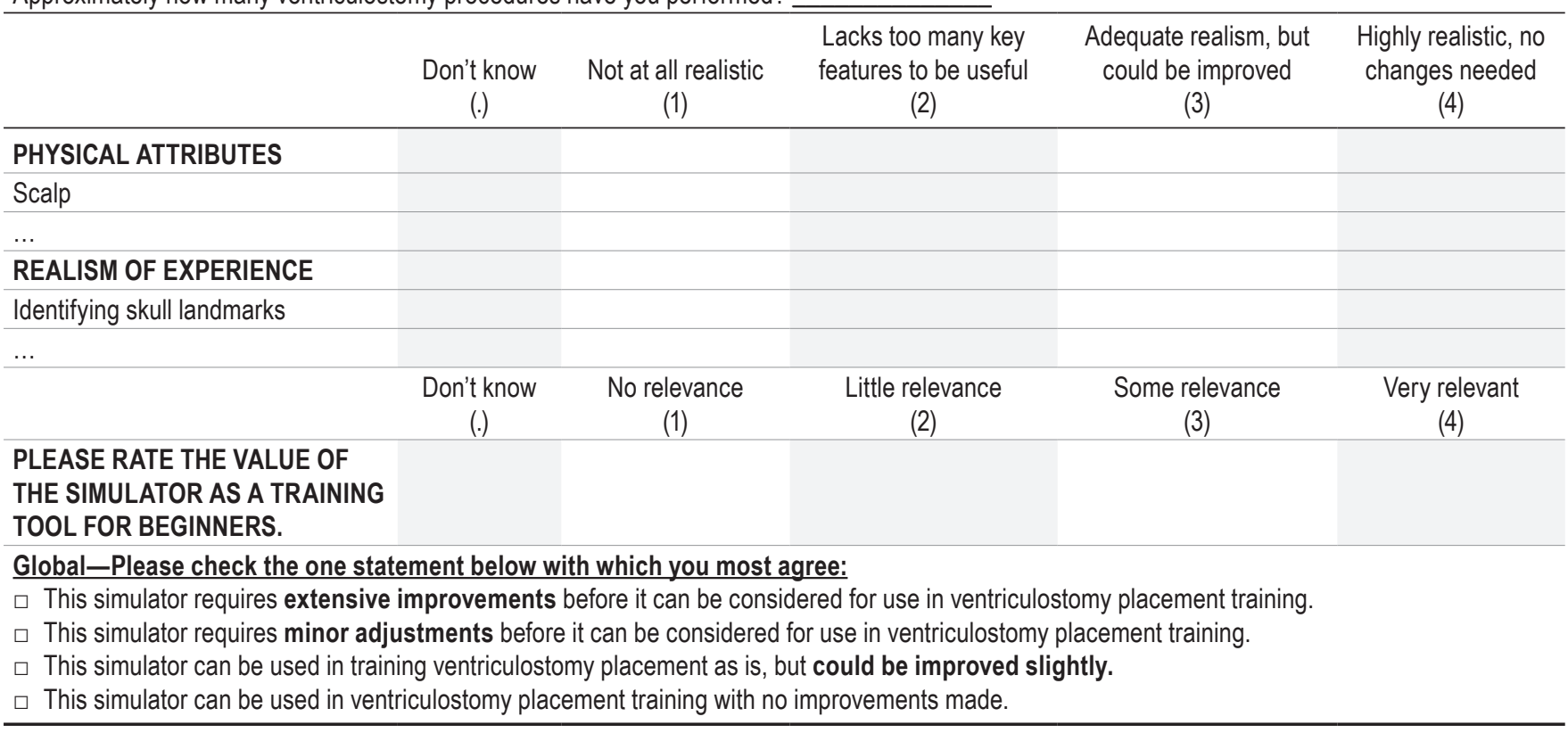



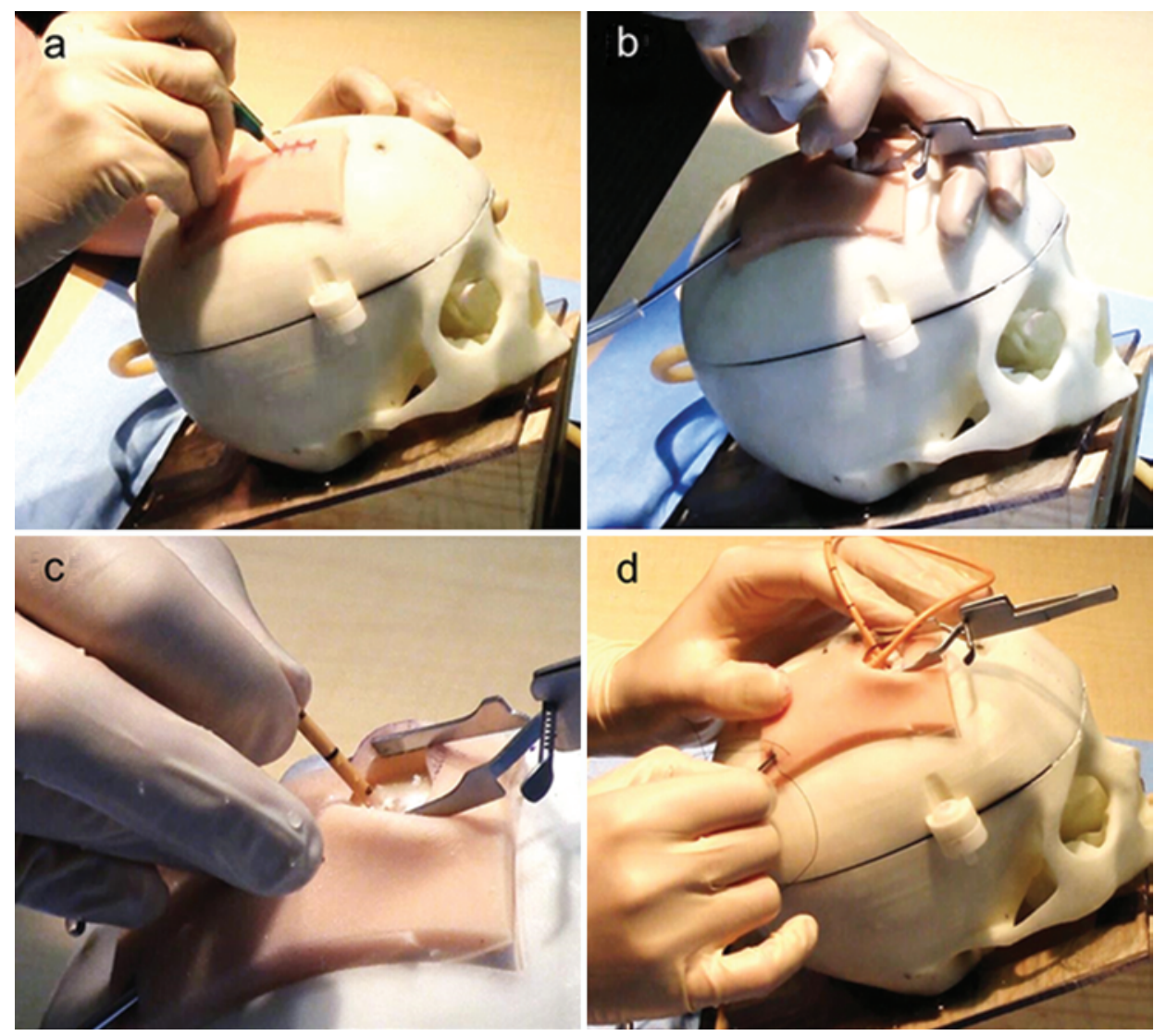

FIG. 5. Images from a representative EVD simulator evaluation showing scalp incision (a), bone drilling (b), catheter placement (c), and tunneling and suturing (d). Figure is available in color online only.

the novice operator to become familiar with the surgical instruments needed to place an EVD, which generally varies among institutions. Finally, our physical simulator has all the necessary features for training in ICP monitor placement, including an intracranial compartment pressurized by a height-adjustable water reservoir. With the flexibility of 3D printing, modifications for distorted anatomy or patient-specific features can be added to the current model.

Manufacturing costs and ancillary technology required to use the existing VR ventriculostomy simulators is a major concern, particularly for the initial investment, which ranges from several tens to hundreds of thousands of US dollars. ${ }^{21}$ The manufacturing cost of a physical EVD simulator is significantly lower (estimated to be within \$1000), and it can be used extensively by replacing the multilayer insert after each use, and the phantom brain after approximately 10 EVD placements. The brain phantom can hold its integrity for 10 or more passes because of the water pressure and material used. Catheter insertion is also not affected by the previous pathways created inside the brain phantom. This feature turns our simulator into a very costeffective training tool, and it is easily accessible to training programs in third-world countries.

Although favorable, preliminary findings indicate areas of the simulator that could be improved prior to implementation into a curriculum. All comments received from the evaluators can be addressed in a refined model. Spe- cifically, dura thickness can be adjusted by the number of layers of the membrane material, and skin resistance can be reduced by mixing 5\%-10\% mineral oil into Dragon Skin silicone (Smooth-on Inc.). ${ }^{24}$ Additional landmarks, such as ears, can be produced by 3D printing onto the existing model. The survey is strongly favorable, but there are a number of limitations that should be considered at this time. First, although the study was multiinstitutional, data were collected from a convenience sample of volunteer participants in southeastern Michigan. Second, the sample evaluated to date is relatively small and may not reflect the opinions of a larger sample. Further research will clarify if the feedback from these evaluators is different from those of a broader sample of neurosurgeons across a variety of regions. Finally, we have only examined preliminary validity evidence from this prototype model, and further simulator refinement and deeper evaluation of different kinds of validity evidence will be required to maximize the fidelity of our simulator in the training of EVD placement. Our future line of research includes evaluating the simulator's utility for measuring surgeon EVD performance, or to better define mastery of the procedure by distinguishing novice from proficient practitioners. Next, a performance rating instrument will need to be created to align all steps of the EVD with a global rating matrix using the Objective Structured Assessment of Technical Skill (OSATS) tool. ${ }^{16}$ Based on those findings, training protocols and evaluation standards, such as the minimum 
TABLE 2. Survey results from 17 experienced neurosurgeons and residents

\begin{tabular}{|c|c|c|}
\hline Survey Items & Mean Score (SD) & Major Comments \\
\hline \multicolumn{3}{|l|}{ Physical attributes } \\
\hline Scalp & $3.1(0.44)$ & Skin is rubbery \\
\hline Bone & $3.4(0.62)$ & \\
\hline Dura thickness & $2.9(0.71)$ & Dura is a little too tough \\
\hline Brain tissue texture & $3.4(0.72)$ & \\
\hline Visualization of skull landmarks & $3.0(0.72)$ & Additional landmarks (ears) are needed \\
\hline Location of insert for drilling & $3.6(0.50)$ & \\
\hline Size of the insert for drilling & $3.9(0.47)$ & Insert could be a little larger anteriorly \\
\hline CSF flow (rate) to confirm placement & $3.6(0.77)$ & \\
\hline \multicolumn{3}{|l|}{ Realism of experience } \\
\hline Identifying skull landmarks & $3.2(0.55)$ & Needs skin landmarks in addition to bone \\
\hline Incision and retraction of skin & $3.1(0.87)$ & \\
\hline Drilling bone & $3.3(0.84)$ & \\
\hline Opening dura & $2.6(0.95)$ & \\
\hline Inserting ventriculostomy & $3.3(0.69)$ & \\
\hline $\begin{array}{l}\text { Water flowing from catheter \& visual confirmation of } \\
\text { trajectory }\end{array}$ & $3.4(0.79)$ & \\
\hline Tunneling of ventriculostomy & $3.4(0.62)$ & Tunneling resistance is not so realistic \\
\hline Placement of retaining stitches & $3.7(0.50)$ & \\
\hline Scalp closure & $3.5(0.52)$ & \\
\hline Value of simulator as a training tool & $3.9(0.25)$ & \\
\hline Global rating & $2.5(0.48)$ & $\begin{array}{l}\text { This simulator requires minor adjustments before it can be considered } \\
\text { for use in ventriculostomy placement training }\end{array}$ \\
\hline
\end{tabular}

score before a resident can operate on a live patient, will be developed.

\section{Conclusions}

In this paper we present a newly developed physical simulator based on 3D printing and molding technologies for EVD placement and report survey results used to evaluate validity evidence relevant to content validity. Survey results from 17 surgeons with a range of expertise strongly support the use of this model for EVD training. With minor refinement, this simulator is expected to improve training experiences in neurosurgery, thereby leading to better patient care. The capability to measure and improve an individual's performance has not yet been fully studied. Following the recommended refinements and future study, we ultimately expect to incorporate this simulator into a comprehensive curriculum to train residents in EVD placement.

\section{Acknowledgments}

We would like to thank the Department of Neurosurgery at both Henry Ford Hospital and Wayne State University for participating in the survey.

\section{References}

1. Alaraj A, Tobin MK, Birk DM, Charbel FT: Simulation in neurosurgery and neurosurgical procedures, in Levine AI, DeMaria S Jr, Schwartz AD, Sim AJ (eds): The Compre- hensive Textbook of Healthcare Simulation. New York: Springer, 2013, pp 415-423

2. American Educational Research Association: Standards for Educational and Psychological Testing. Washington, DC: American Educational Research Association, 2014

3. Banerjee PP, Luciano CJ, Lemole GM Jr, Charbel FT, Oh MY: Accuracy of ventriculostomy catheter placement using a head- and hand-tracked high-resolution virtual reality simulator with haptic feedback. J Neurosurg 107:515-521, 2007

4. Bova FJ, Rajon DA, Friedman WA, Murad GJ, Hoh DJ, Jacob RP, et al: Mixed-reality simulation for neurosurgical procedures. Neurosurgery 73 (Suppl 1):138-145, 2013

5. Bridges M, Diamond DL: The financial impact of teaching surgical residents in the operating room. Am J Surg 177:2832, 1999

6. Bryson EO, Levine AI: The simulation theater: a theoretical discussion of concepts and constructs that enhance learning. J Crit Care 23:185-187, 2008

7. Chatzi M, Karvouniaris M, Makris D, Tsimitrea E, Gatos $\mathrm{C}$, Tasiou A, et al: Bundle of measures for external cerebral ventricular drainage-associated ventriculitis. Crit Care Med 42:66-73, 2014

8. Chen RK, Shih AJ: Multi-modality gellan gum-based tissuemimicking phantom with targeted mechanical, electrical, and thermal properties. Phys Med Biol 58:5511-5525, 2013

9. Guyot LL, Dowling C, Diaz FG, Michael DB: Cerebral monitoring devices: analysis of complications. Acta Neurochir Suppl 71:47-49, 1998

10. Hoefnagel D, Dammers R, Ter Laak-Poort MP, Avezaat CJ: Risk factors for infections related to external ventricular drainage. Acta Neurochir (Wien) 150:209-214, 2008

11. Huyette DR, Turnbow BJ, Kaufman C, Vaslow DF, Whiting BB, Oh MY: Accuracy of the freehand pass technique for ventriculostomy catheter placement: retrospective assessment 
using computed tomography scans. J Neurosurg 108:88-91, 2008

12. Lemole GM Jr, Banerjee PP, Luciano C, Neckrysh S, Charbel FT: Virtual reality in neurosurgical education: part-task ventriculostomy simulation with dynamic visual and haptic feedback. Neurosurgery 61:142-149, 2007

13. Lemole M, Banerjee PP, Luciano C, Charbel F, Oh M: Virtual ventriculostomy with 'shifted ventricle': neurosurgery resident surgical skill assessment using a high-fidelity haptic/ graphic virtual reality simulator. Neurol Res 31:430-431, 2009

14. Luciano C, Banerjee P, Lemole GM Jr, Charbel F: Second generation haptic ventriculostomy simulator using the ImmersiveTouch system. Stud Health Technol Inform 119:343-348, 2006

15. Maniker AH, Vaynman AY, Karimi RJ, Sabit AO, Holland B: Hemorrhagic complications of external ventricular drainage. Neurosurgery 59 (4 Suppl 2):ONS419-ONS425, 2006

16. Martin JA, Regehr G, Reznick R, MacRae H, Murnaghan J, Hutchison C, et al: Objective structured assessment of technical skill (OSATS) for surgical residents. Br J Surg 84:273278, 1997

17. Moon HH, Kim JH, Kang HI, Moon BG, Lee SJ, Kim JS: Brain injuries during intraoperative ventriculostomy in the aneurysmal subarachnoid hemorrhage patients. J Korean Neurosurg Soc 46:215-220, 2009

18. Okuda Y, Bryson EO, DeMaria S Jr, Jacobson L, Quinones J, Shen B, et al: The utility of simulation in medical education: what is the evidence? Mt Sinai J Med 76:330-343, 2009

19. Puram SV, Kozin ED, Sethi R, Alkire B, Lee DJ, Gray ST, et al: Impact of resident surgeons on procedure length based on common pediatric otolaryngology cases. Laryngoscope 125:991-997, 2015

20. Roitberg BZ, Khan N, Alp MS, Hersonskey T, Charbel FT, Ausman JI: Bedside external ventricular drain placement for the treatment of acute hydrocephalus. Br J Neurosurg 15:324-327, 2001

21. Schirmer CM, Elder JB, Roitberg B, Lobel DA: Virtual reality-based simulation training for ventriculostomy: an evidence-based approach. Neurosurgery 73 (Suppl 1):66-73, 2013

22. Schroeder HW, Niendorf WR, Gaab MR: Complications of endoscopic third ventriculostomy. J Neurosurg 96:10321040, 2002

23. Seagull FJ, Rooney DM: Filling a void: developing a standard subjective assessment tool for surgical simulation through focused review of current practices. Surgery 156:718-722, 2014

24. Wang Y, Tai BL, Yu H, Shih AJ: Silicone-based tissuemimicking phantom for needle insertion simulation. J Med Devices 8:021001, 2014

25. Waran V, Narayanan V, Karuppiah R, Owen SL, Aziz T: Utility of multimaterial 3D printers in creating models with pathological entities to enhance the training experience of neurosurgeons. J Neurosurg 120:489-492, 2014

26. Wilson T, Sahu A, Johnson DS, Turner PG: The effect of trainee involvement on procedure and list times: A statistical analysis with discussion of current issues affecting orthopaedic training in UK. Surgeon 8:15-19, 2010

\section{Author Contributions}

Conception and design: Tai, Shih, Savastano. Acquisition of data: Tai, Stephenson, Liao, Savastano. Analysis and interpretation of data: Tai, Rooney. Drafting the article: Tai, Savastano. Critically revising the article: Sagher, Tai. Reviewed submitted version of manuscript: all authors. Approved the final version of the manuscript on behalf of all authors: Sagher. Administrative/technical/ material support: Stephenson, Liao. Study supervision: Sagher, Tai, Shih.

\section{Correspondence}

Oren Sagher, Department of Neurosurgery, University of Michigan, 1500 E. Medical Center Dr., Rm. 3552 TC, Ann Arbor, MI 48109-5338. email: osagher@med.umich.edu. 